\title{
Nanoscale dynamics in complex materials by resonant X-ray Photon Correlation Spectroscopy (rXPCS)
}

\author{
Alessandro Ricci* \\ Deutsches Elektronen-Synchrotron DESY, Notkestraße 85, D-22607 Hamburg, Germany \\ * alessandro.ricci@desy.de \\ *phd.alessandro.ricci@gmail.com
}

\begin{abstract}
Complex materials are characterized by a competition between multiple phases that coexist in a nanoscale phase separation scenario. In particular there is a growing interest in understanding how the competition between Charge-Density-Wave (CDW), the Spin-Density-Wave (SDW) and the defects puddles promotes the material's functionality at the macroscopic scale. For this reason the finding of a new technique that could combine temporal and spatial resolution with bulk sensitivity, is extremely important. A good solution could arrive by the use of a time-resolved scattering technique like X-ray Photon Correlation Spectroscopy (XPCS). As example of possible application we propose the study of

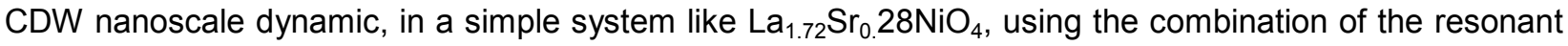
X-ray Scattering (RXS) and XPCS. This could provide important information on the CDW nanoscale dynamic in complex material characterized by nanoscale phase separation.
\end{abstract}

The physics of complex materials and the emerging coherent macroscopic state like superconductivity is still an open problem [1,2]. In particular, the interplay between the Charge-Density-Wave (CDW), the SpinDensity-Wave (SDW) and the defects ordering is under an intense discussion [3,4]. Indeed there is a strong interest in understanding how the competition, between these phases forming puddles, plays a key role in the promotion of the material's functionality at the macroscopic scale.

It is known that the macroscopic properties of complex materials can be tuned by external fields or temperature [5]. These processes occur not via a complete phase transition but, by a spatial rearrangement of the puddles of different coexisting phases. For example the macroscopic metallic behavior, can be obtained via a percolation of metallic domains embedded into an insulating matrix. Even the colossal magnetoresistance (CMR) has been predicted to be due by the competition between different phases forming a nanoscale phase separation scenario [6].

In order to clarify the phase's coexistence it is important to understand their spatial organization. Up to now only few works point the attention to the visualization of how SDW, CDW and defects puddles are organized. Moreover most of them just probe the local electronic structure using scanning probe techniques [7] or absorption spectroscopy [8-10], which are limited to the surface layer only, whose properties may deviate from the bulk considerably. On this purpose an important solution came from the use of innovative techniques like scanning micro X-ray diffraction ( $\mathrm{S} \mu \mathrm{XRD})$ and resonant scanning micro 
X-ray diffraction ( $r S \mu X R D)$ that allow to directly visualize the bulk spatial organization of the SDW, CDW and defects puddles. Indeed by the use of $S \mu X R D$ on several High temperature superconductors (HTS) it has been possible to directly visualize a complex nanoscale phase separation characterized by the coexistence of competing granular networks of different puddles [11-21] forming a complex organization like in social networks [22]. The use of $S \mu X R D$ has even allowed to demonstrate that an optimum inhomogeneity is needed to promote the percolation of one of the present phases in the material [23] characterized by a critical misfit-strain [24-26]. Even if there is now a converging agreement on the spatial complex organization of multiple phases, the way how they evolve in time is still a fundamental missing point for the explanation of the percolation process. Indeed the available time-resolved reflectivity measurements [27], probe mainly the macroscopic properties and they are not sensitive to spatial inhomogeneities. For this reason there is a strong need to find a new technique that could combine temporal and spatial resolution with bulk sensitivity. A good solution could arrive by the use of a timeresolved scattering technique like X-ray Photon Correlation Spectroscopy (XPCS). XPCS is a coherent Xray scattering technique, which allows to investigate the dynamic behavior of materials on nanometers length scale [28-31]. It has been successfully applied to study the time equilibrium or quasi-equilibrium dynamic in a broad variety of soft complex materials like colloidal gels and glasses [32-35], complex liquids [36], and to investigate fluctuation in biomaterials [37-38]. Recently, this versatile technique has been also used for the characterization of nanoparticles dynamics in suspension [39] and on thin polymer films [40]. On the other hand, the application of XPCS to the study of solids has not developed too much, even if interesting results have been obtained investigating atomic diffusion process in metals $[41,42]$, antiferromagnetic domains fluctuation [43,44], slow dynamics in alloys [45-49] and avalanches in martensitic materials [50-52]. Nevertheless the bulk sensitivity of XPCS and its capability to combine temporal and spatial resolution make this technique particularly suitable for the investigation of dynamic behavior of complex materials where multiple phases coexist. In a typical XPCS experiment a microfocused coherent beam is used to collect a sequence of speckle patterns and the intensity autocorrelation function $g_{2}$ is used to determine the correlation-time $\tau$ at each wavevector $\mathrm{Q}$ :

$$
g_{2}(t)=\frac{\langle I(\tau) I(\tau+t)\rangle}{\langle I(\tau)\rangle^{2}}=1+A|F(Q, t)|^{2}
$$

With this method the dynamics can be characterized on timescales as short as the time spacing $\Delta t$ of the sequence. Under certain conditions this function allows access to the intermediate scattering function: $|F(Q, t)|$, which can be modeled by theory. The most used model for describing equilibrium dynamic is the Kohlrausch-Williams-Watts (KWW) model, consisting in an exponential form: $|F(Q, t)|=\exp \left(-\left({ }^{t} / \tau\right)^{\beta}\right)$ where $\tau$ is the characteristic time scale of the dynamic and $\beta$ is the critical exponent.

The information about the time and length scales of domain dynamics is extremely important to clarify how different phases like CDW, SDW and defects organization, compete in the same material in a nanoscale phase separation scenario [16]. The deep understanding of the microscopic processes that govern this phenomenon has the potential to allow for controlling and developing novel functional materials. 
Moreover, to study the nanoscale dynamics of the CDW and SDW domains it is possible to combine XPCS with the resonant X-ray Scattering technique (RXS) obtaining a new useful tool: the resonant X-ray Correlation Spectroscopy ( $\mathrm{XPCS}$ ). In a rXPCS experiment, in order to increase the sensitivity to magnetic states or bands, the incident beam energy is tuned to distinct absorption edges in the material. $\mathrm{M}_{4,5}$ and $L_{2,3}$ absorption edges can be selected to investigate rare-earth systems and $3 d$ transition-metals, respectively. Circularly polarised and soft $\mathrm{x}$-rays could be also useful for specific layouts. rXPCS experiments allow the study of CDW and SDW domains dynamic down to domain sizes of several nm in $3 d$ transition-metals, exploiting $2 p-3 d$ scattering resonances, and in rare-earth compounds, exploiting the 3d-4f processes.

An interesting application of rXPCS could be the study of the CDW nanoscale dynamic in $\mathrm{La}_{1.72} \mathrm{Sr}_{0.28} \mathrm{NiO}_{4}$. In this complex material a magnetic Bragg peak has been observed at the wave vector $(2 \varepsilon 00)$ where the incommensurability $\varepsilon=0.298$ is inversely proportional to the CDW stripes period. A strong resonant enhancement of the magnetic reflections can be observed tuning the energy at the Ni L3-edge (855 eV) like is shown in Figure $1 \mathrm{~A}$. Using the micro-focused high-coherent beam available at the new generation synchrotron facilities would be possible to follow the time speckles evolution of the resonant CDW peak (Figure 1B). The speckle patterns change in time (Figure 1C) and the intensity autocorrelation function $g_{2}$ (Figure $1 \mathrm{D}$ ) can be calculated to determine the characteristic relaxation-time $\tau$ of the dynamic. Moreover, cooling down to $20 \mathrm{~K}$ with a helium-cryostat the peak intensity would be strongly enhanced allowing studying the CDW nanoscale dynamic as a function of temperature.

\section{Conclusion}

A short overview of the use of X-ray Photon Correlation Spectroscopy is presented. Resonant XPCS, is suggested as a possible powerful technique to put light on the controversial problem of phases competition in complex materials. On this purpose, we propose the study of CDW domains fluctuations using the combination of the RXS to visualize CDW structure and XPCS to study the CDW domains fluctuations in time as a function of temperature. The charge-ordered system $\mathrm{La}_{1.72} \mathrm{Sr}_{0.28} \mathrm{NiO}_{4}$, would be a simple model to understand the phase separation scenario that characterize complex materials where multiple phases of ordered charges, spins and lattice stripes compete to promote emerging properties at the macroscopic scale. This would be the first observation of nanoscale dynamic of the CDW domains in a complex material characterized by nanoscale phase separation. To have clear information about the length and time scales of phase separation effect could provide extremely important new inputs for the theoretical modeling of microscopic process that promote emerging properties in functional complex materials. 
Figures
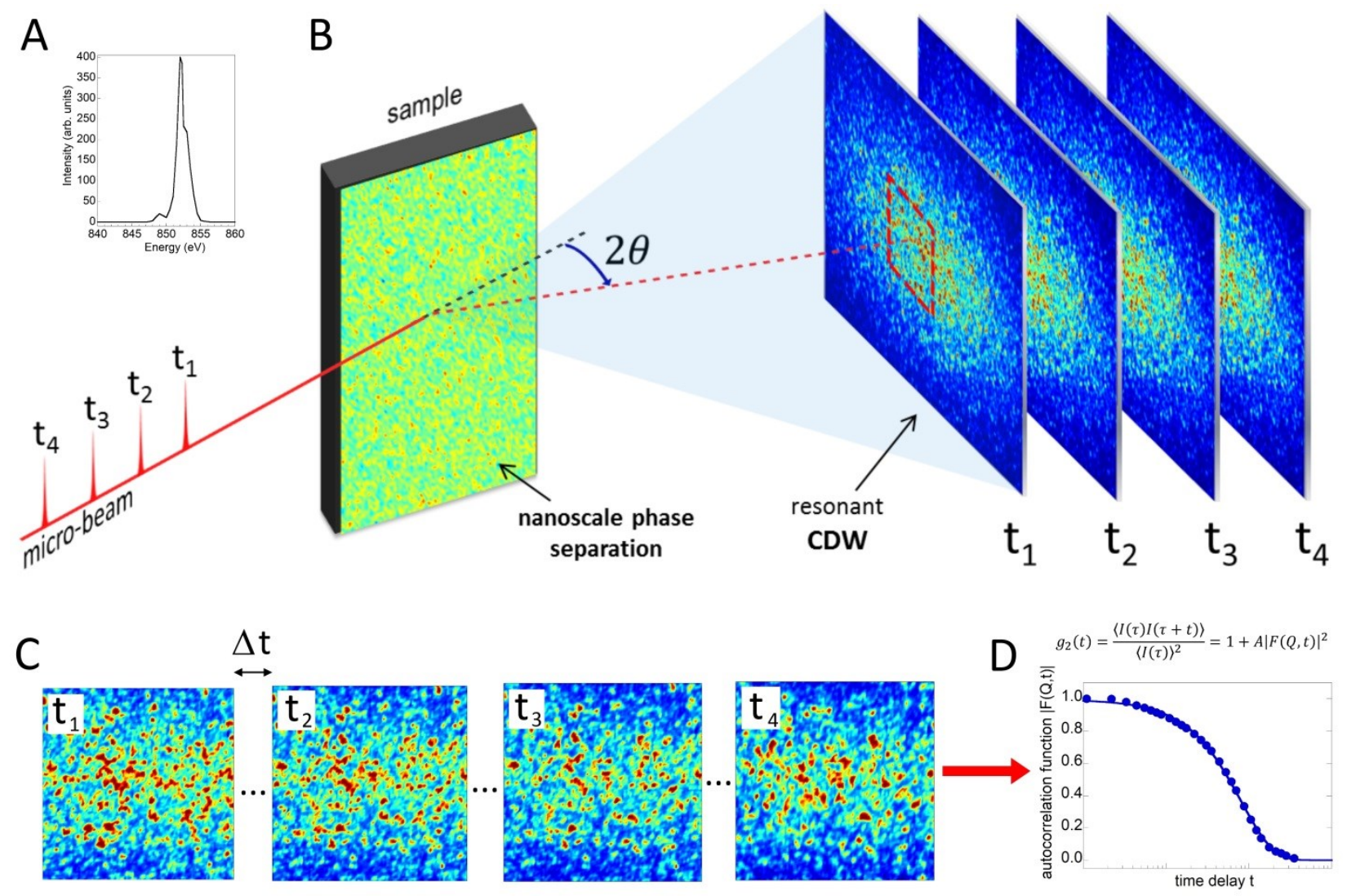

Figure 1: resonant X-ray Photon Correlation Spectroscopy experiment.

Panel A: the integrated intensity of resonant CDW peak in $\mathrm{La}_{1.72} \mathrm{Sr}_{0.28} \mathrm{NiO}_{4}$ as a function of energy. The scattering intensity is strongly enhanced setting the energy to the $\mathrm{Ni}_{3}$-edge.

Panel B: A sample showing nanoscale phase separation is illuminated by a coherent x-ray micro-beam. A time series of diffraction patterns is collected keeping external condition as stable as possible.

Panel C: The time-dependent coherent diffraction patterns are characterized by the presence of speckles that represent the granular local structure of the material showing an inhomogeneous landscape of charge-ordered domains in the illuminated spot.

Panel D: In order to determine the correlation-time $\tau$ that characterizes the CDW domains dynamic, the intensity autocorrelation function can be calculated. This would allow to probe dynamics on timescales as short as the time spacing $\Delta t$ of the collected time series.

\section{Acknowledgements}

The author wants to dedicate the work to the fond memory of the late Professor Joseph Ashkenazi. 


\section{References}

[1] J. Ashkenazi, N.F. Johnson, Journal of Superconductivity and Novel Magnetism, 26, 2611 (2013).

[2] J. Ashkenazi, Journal of Superconductivity and Novel Magnetism, 24, 1281 (2011).

[3] A. Bianconi, Nature Physics 9, 536 (2013), ISSN 1745-2473

[4] G. Ghiringhelli, M. Le Tacon, M. Minola, S. Blanco-Canosa, C. Mazzoli, N. B. Brookes, G. M. De Luca, A. Frano, D. G. Hawthorn, F. He, et al., Science 337, 821 (2012), ISSN 1095-9203

[5] M. Imada, A. Fujimori, Y. Tokura, Rev. Mod. Phys. 70, 1039 (1998).

[6] E. Dagotto, Science 309, 257 (2005).

[7] T. Becker, C. Streng, Y. Luo, V. Moshnyaga, B. Damaschke, N. Shannon, and K. Samwer, Physical review letters 89.23 (2002): 237203.

[8] A. Bianconi, S. Della Longa, C. Li, M. Pompa, A. Congiu-Castellano, D. Udron, A Flank, P. Lagarde, Physical Review B 44 (18), 10126-10138 (1991).

[9] A. Bianconi, M. Missori, H. Oyanagi, H. Yamaguchi, Y. Nishiara, S. Della Longa, Europhysics Letters 31 (7), 411-415 (1995).

[10] A. Lanzara, N.L. Saini, M. Brunelli, A. Valletta, A. Bianconi, Journal of Superconductivity and Novel Magnetism 10 (4), 319-321 (1997)

[11] M. Fratini, N. Poccia, A. Ricci, G. Campi, M. Burghammer, G. Aeppli, and A. Bianconi, Nature 466, 841 (2010), ISSN 0028-0836

[12] N. Poccia, M. Fratini, A. Ricci, G. Campi, L. Barba, A. Vittorini-Orgeas, G. Bianconi, G. Aeppli, and A. Bianconi, Nature Materials 10, 733 (2011), ISSN 1476-1122

[13] N. Poccia, M. Chorro, A. Ricci, W. Xu, A. Marcelli, G. Campi, and A. Bianconi, Applied Physics Letters 104, 221903+ (2014), ISSN 0003-6951

[14] N. Poccia, A. Bianconi, G. Campi, M. Fratini, and A. Ricci, Superconductor Science and Technology 25, 124004+ (2012), ISSN 0953-2048

[15] N. Poccia, G. Campi, M. Fratini, A. Ricci, N. L. Saini, and A. Bianconi, Physical Review B 84, 100504+ (2011), ISSN 1098-0121

[16] A. Ricci, N. Poccia, G. Campi, B. Joseph, G. Arrighetti, L. Barba, M. Reynolds, M. Burghammer, H. Takeya, Y. Mizuguchi, et al., Physical Review B 84, 060511+ (2011), ISSN 1098-0121

[17] D. Innocenti, A. Ricci, N. Poccia, G. Campi, M. Fratini, and A. Bianconi, Journal of Superconductivity and Novel Magnetism 22, 529 (2009), ISSN 1557-1939

[18] A. Ricci, N. Poccia, B. Joseph, G. Arrighetti, L. Barba, J. Plaisier, G. Campi, Y. Mizuguchi, H. Takeya, Y. Takano, et al., Superconductor Science and Technology 24, 082002+ (2011)

[19] G. Campi, A. Ricci, N. Poccia, L. Barba, G. Arrighetti, M. Burghammer, A. S. Caporale, and A. Bianconi, Physical Review B 87, 014517+ (2013)

[20] A. Ricci, N. Poccia, G. Campi, F. Coneri, A. S. Caporale, D. Innocenti, M. Burghammer, M. Zimmermann, and A. Bianconi, Scientific Reports 3, 2383+ (2013), ISSN 2045-2322 
[21] A. Ricci, N. Poccia, G. Campi, F. Coneri, L. Barba, G. Arrighetti, M. Polentarutti, M. Burghammer, M. Sprung, Martin, et al., New Journal of Physics 16, 053030+ (2014), ISSN 1367-2630

[22] N. F. Johnson, J. Ashkenazi, Z. Zhao, L. Quiroga, arXiv preprint arXiv:1011.6398 (2010).

[23] N. Poccia, A. Ricci, G. Campi, M. Fratini, A. Puri, D. Di Gioacchino, A. Marcelli, M. Reynolds, M. Burghammer, N. L. Saini, et al., Proceedings of the National Academy of Sciences 109, 15685 (2012)

[24] N. Poccia, A. Ricci, A. Bianconi, Advances in Condensed Matter Physics (2010)

[25] A. Ricci, N. Poccia, G. Ciasca, M. Fratini, and A. Bianconi, Journal of Superconductivity and Novel Magnetism 22, 589 (2009), ISSN 1557-1939

[26] A. Ricci, M. Fratini, and A. Bianconi, Journal of Superconductivity and Novel Magnetism 22, 305 (2009), ISSN 1557-1939

[27] D. Polli, M. Rini, S. Wall, R. W. Schoenlein, Y. Tomioka, Y. Tokura, G. Cerullo, and A. Cavalleri, Nature Materials 6, 643 (2007), ISSN 1476-1122

[28] G. Grübel \& F. Zontone (2004). 'Correlation spectroscopy with coherent X-rays'. Journal of Alloys and Compounds 362(1-2):3-11.

[29] G. Grübel, et al. (2007). 'XPCS at the European X-ray free electron laser facility'. Nuclear Instruments and Methods in Physics Research Section B: Beam Interactions with Materials and Atoms 262(2):357-367.

[30] G. Grübel, et al. (2008). 'X-Ray Photon Correlation Spectroscopy (XPCS)'. In R. Borsali \& R. Pecora (eds.), Soft Matter Characterization, pp. 953-995. Springer Netherlands.

[31] A. Madsen, et al. (2010). 'Beyond simple exponential correlation functions and equilibrium dynamics in x-ray photon correlation spectroscopy'. New Journal of Physics 12(5):055001+.

[32] Fluerasu, et al. (2007). 'Slow dynamics and aging in colloidal gels studied by x-ray photon correlation spectroscopy'. Physical Review E 76(1).

[33] T. Thurn-Albrecht, et al. (1996). 'Photon Correlation Spectroscopy of Colloidal Palladium Using a Coherent X-Ray Beam'. Physical Review Letters 77(27):5437-5440.

[34] H. Grigoriew, et al. (2010). 'Dynamic correlation in magnetorheological composite under magnetic field studied by XPCS'. Solid State Communications 150(17-18):840-843.

[35] D. Pontoni, et al. (2003). 'Microstructure and Dynamics near an Attractive Colloidal Glass Transition'. Physical Review Letters 90(18).

[36] S. O. Hruszkewycz, et al. (2012). 'High Contrast X-ray Speckle from Atomic-Scale Order in Liquids and Glasses'. Physical Review Letters 109:185502+.

[37] Sikharulidze, et al. (2002). 'Smectic Membranes in Motion: Approaching the Fast Limits of X-Ray Photon Correlation Spectroscopy'. Physical Review Letters 88:115503+.

[38] Sikharulidze, et al. (2003). 'Surface and Bulk Elasticity Determined Fluctuation Regimes in Smectic Membranes'. Physical Review Letters 91:165504+.

[39] A. Robert, et al. (2005). 'Structure and dynamics of electrostatically interacting magnetic nanoparticles in suspension'. The Journal of Chemical Physics 122(8):084701+. 
[40] S. Streit, et al. (2007). 'Two-Dimensional Dynamics of Metal Nanoparticles on the Surface of Thin Polymer Films Studied with Coherent X Rays'. Physical Review Letters 98(4):047801+.

[41] M. Leitner, et al. (2009). 'Atomic diffusion studied with coherent X-rays'. Nat Mater 8(9):717-720.

[42] G. B. Stephenson, et al. (2009). 'X-ray spectroscopy: Revealing the atomic dance'. Nat Mater 8(9):702-703.

[43] O. G. Shpyrko, et al. (2007). 'Direct measurement of antiferromagnetic domain fluctuations'. Nature 447(7140):68-71.

[44] S. Konings, et al. (2011). 'Magnetic Domain Fluctuations in an Antiferromagnetic Film Observed with Coherent Resonant Soft X-Ray Scattering'. Physical Review Letters 106(7):077402+.

[45] S. Brauer, et al. (1995). 'X-Ray Intensity Fluctuation Spectroscopy Observations of Critical Dynamics in Fe3Al'. Physical Review Letters 74(11):2010-2013.

[46] J. A. Pitney, et al. (2000). 'Streaked speckle in Cu3Au coherent x-ray diffraction'. Physical Review B 62:13084-13088.

[47] L.-M. Stadler, et al. (2004). 'Revealing antiphase-domain dynamics in alloys by combining advanced statistical techniques with x-ray photon correlation spectroscopy'. Physical Review B 69(22).

[48] L.-M. Stadler, et al. (2005). 'Investigating slow dynamics in alloys using X-ray photon correlation spectroscopy'. Nuclear Instruments and Methods in Physics Research Section B: Beam Interactions with Materials and Atoms 238(1-4):189-191.

[49] A. Fluerasu, et al. (2005). 'X-Ray Intensity Fluctuation Spectroscopy Studies on Phase-Ordering Systems'. Physical Review Letters 94(5).

[50] L. Müller, et al. (2006). 'Time-dependent phenomena in athermal martensitic transformations'. Materials Science and Engineering: A 438-440:122-125.

[51] L. Müller, et al. (2011). 'Slow Aging Dynamics and Avalanches in a Gold-Cadmium Alloy Investigated by X-Ray Photon Correlation Spectroscopy'. Physical Review Letters 107:105701+.

[52] B. Ruta, et al. (2012). 'Atomic-Scale Relaxation Dynamics and Aging in a Metallic Glass Probed by X-Ray Photon Correlation Spectroscopy'. Physical Review Letters 109:165701+. 